\title{
An ihren Gedanken, nicht ihren Taten sollt ihr sie erkennen!
}

Ralf Kölbel und Susanne Selter

\begin{abstract}
Diogenes hielt jedem, der ihm auf dem Marktplatz von Athen begegnete, eine Leuchte ins Gesicht, um festzustellen, ob er es mit einem ehrlichen Menschen zu tun habe. „O.K.“, mögen wir sagen, Diogenes war sowieso ein „Freak“ und offenbar auch paranoid. Doch halt, bevor unser Urteil allzu schonungslos ausfällt: Sind wir nicht ebenso bemüht zu erfahren, wer mit welchem Ansinnen unter uns ist? Fürchten nicht gerade wir uns vor allem und jedem - nämlich nicht nur vor Krankheit und Unglück, sondern auch vor dem Bösen (Terroristen, Mafiosi, Perversen ....)? ${ }^{1}$ Und greifen wir deshalb nicht zu sämtlichen Methoden, von denen wir uns Schutzeffekte versprechen? Womöglich werden wir damit auch nicht einhalten wollen, falls sich hierzulande eine technisch ganz neue, auch qualitativ neue (!) Maßnahme etabliert, die - natürlich in guter präventiver Absicht - endlich den kriminellen Antrieb aufzudecken verheißt: den feindlichen Willen. Gemeint ist ein aufschlussreiches Verfahren: ein Gedanken lesendes „Mind-Screening“ erstaunlicher Art.
\end{abstract}

\section{Die Logik des Risikostaates: Vorbeugen statt Kontern}

„Zum Schutz der Inneren Sicherheit“, wie wir es nennen, hat sich das Staatswesen des Westens schleichend verändert. Man hat das hinreichend beschrieben und an zahlreichen Facetten belegt ${ }^{2}$. Jener Prozess wird unter anderem dort augenfällig, wo sich Ermittlungs- und Eingriffsbefugnisse nicht nur beständig vermehren, sondern unversehens auch in ihrer Zielrichtung wandeln: Unter dem Label der „Vorfeldermittlung“ (bzw. des „proaktiven Polizeihandelns“ bzw. der „vorbeugenden Straftatbekämpfung ") fahndet die Polizei anlassunabhängig nach Ermittlungsanstößen und -gründen ${ }^{3}$. Hiermit greift, gemessen an den klassischen Strategien der Aufklärung konkreter Verdachtslagen und der Abwehr konkreter Gefahren, eine Handlungslogik um sich, die man lange Zeit den Nachrichtendiensten vorbehielt ${ }^{4}$.

Erklärbar ist diese Entwicklung durch das Bestreben, ein wie auch immer geartetes Risiko - gleichviel, wie sehr dieses realiter besteht und/oder durch den gesellschaftlichen Diskurs nur suggeriert ist ${ }^{5}-$ zu bekämpfen. Als Risiko versteht sich dabei die statistische
Wahrscheinlichkeit des Schadenseintritts ${ }^{6}$, die zwar durch Einzelanlässe in das Bewusstsein gerückt werden mag, hernach aber eher als unheilvolle, diffuse Drohung dauerhaft fortexistiert. Als deren Quelle erscheinen dann die „gefährlichen Anderen“ als typischerweise de-individualisierte Struktur (aktuell: „der islamistische Terrorismus“). Dadurch wird ein gesteigertes Sicherheitsbedürfnis ausgelöst, das nach möglichst frühzeitiger Risikoerkennung und -eindämmung verlangt ${ }^{7}$. Maßnahmen, mit denen der Staat dem zu entsprechen versucht ${ }^{8}$, stellen dieses Begehren freilich niemals zufrieden - und dies allein schon deshalb, weil das SchutzBemühen die Verunsicherung geradezu reproduziert ${ }^{9}$. Akte der Risikoprävention hören, um Deleuze zu paraphrasieren, nie damit auf anzufangen ${ }^{10}$. Sicherheit bleibt ein ständig erstrebtes, aber unerreichbares Ideal, das immerfort noch maßlosere Anstrengung zeugt. So bringt der Versuch, das Risiko zu beherrschen, eine nie da gewesene Neugier mit sich, die konsequenterweise - um das Unheil schon im Keim zu erkennen -in immer frühere Gefahrenstadien vorstößt.

In Gestalt der polizeilichen „Gefahren- und Straftatenvorsorge" erhält diese Vorgehensweise seit den 1980er Jahren in den Länder-Polizeigesetzen ${ }^{11}$ ihre rechtliche Form. Charakteristisch für diese Maßnahmen, die weit im Schadens-Vorfeld agieren, ist unter anderem, dass ihre Vornahme

- sich einer verdachts- und gefahrenunabhängigen polizeilichen Initiative verdankt (Eingriffs-Anlässe also erst generiert) und

- sich durch Heimlichkeit oder den Zugriff auf umfassende Datensätze fast durchweg in grundrechtssensiblen Bereichen bewegt ${ }^{12}$.

\section{Das Wuchern der Technik: Gedankencheck}

Unserem Gegenüber nicht hinter die Stirn schauen zu können, zählt zu den Basisbedingungen menschlicher Interaktion - und war schon immer zugleich auch ein Stachel, der zur Überwindung der Blickschranken reizt. Deshalb treibt uns ein beständiger Ehrgeiz umher, Auge in Auge zu lesen, was dem anderen ,ins Gesichts geschrieben steht“. Wiewohl diese naive „Hermeneutik“ in un- serer Alltagskultur fest implementiert ist, liegt ihre Irrtumsanfälligkeit klar auf der Hand $^{13}$. Ließe sich diese Fehlerhaftigkeit allerdings mit technischen Mitteln beheben, wäre dies für eine Sicherheitsgesellschaft hoch interessant. Und tatsächlich: Abhilfe scheint derzeit näher denn je.

Als Schlüsselfigur hierbei fungiert Paul Ekman, einer der weltweit angesehensten Experten für nonverbale Kommunikation. Seit mehr als 40 Jahren erforscht er, wie Gefühle entstehen, wie sie sich äußern und erkennen lassen. Ekman wies nach, was zwar Darwin bereits 1872 in „Der Ausdruck der Gemütsbewegungen bei den Menschen und den Tieren" gemutmaßt hatte, was der modernen Forschung jedoch als abwegig galt: die Schemata der emotionalen Mimik werden in allen Kulturkreisen weithin übereinstimmend interpretiert. Beinahe acht Jahre haben Ekman und seine Mitstreiter damit zugebracht, die Anatomie des Gesichts zu erfassen, Gesichtsausdrücke im Bild festzuhalten und in dem sogenannten „Facial Action Coding System“ “(,FACS“) nach den sieben Basisemotionen (Fröhlichkeit, Wut, Ekel, Furcht, Verachtung, Trauer und Überraschung) geordnet zu katalogisieren. Von zehntausend Mienen, die der Mensch dank seiner dreiundvierzig „Action Units“ (d.h. Gesichtsbewegungen durch die verschiedenen Muskeln) zu bilden vermag, filterten sie jene Spielarten heraus, in denen das Repertoire der Gefühle (mit gewissen Variationen) zum Ausdruck gelangt - und von uns prinzipiell gelesen werden kann $^{14}$.

Natürlich bleibt stets das Problem der Verstellung. Allerdings sei es nach Ekman dank der von ihm entdeckten ,micro-momentary facial expressions" prinzipiell lösbar. In diesen Ausdrücken - d.h. jenen blitzschnellen, unbewussten Regungen, die ein falsches Lächeln, eine aufgesetzte Fassade enthüllen und jede Maske brüchig werden lassen - entwickle das Gesicht ein Eigenleben, das sich unserer Kontrolle entzieht. Natürlich schlage sich auf diese Weise kein Denken oder Planen nieder, sondern nur das damit einhergehende Gefüh $1^{15}$. Doch immerhin: Diesen Teil unserer emotionsbestimmten, unwillkürlichen Mimik, der uns für weniger als eine Fünfzehntelsekunde beherrscht, könne man nicht verfälschen ${ }^{16}$. Damit öffne sich für den Beobachter ein Weg hinter die 
Stirn. Selbst, wenn man ohne Übung jene Mikroexpressionen kaum registriert, lasse sich das Gesichterdeutung daher durchaus erlernen.

Die Paul Ekman Group bietet entsprechende Unterweisungen an. Zu den Kunden zählen FBI, CIA und seit 9/11 selbstredend auch das "Department of Homeland Security“, das die Mitarbeiter der „Transport Security Administration“ zur Ausbildung schickt. Statt nur Scheren und Feuerzeuge einzuziehen, sollen sie auf die feindseligen Absichten der Fluggäste achten. Zur Terrorabwehr wurden seit 2003 insgesamt 2800 „Behavior Detection Officers“ in der von Ekman entwickelten „SPOT“-Methode („Screening Passengers through observational Techniques") geschult ${ }^{17}$. Mittlerweile patrouillieren diese professionellen Mienenbeobachter durch die Abfertigungshallen von 50 US-Airports und fischen verräterische Gesichter aus dem Strom der Reisenden heraus. Jeweils paarweise unterwegs, wirken die uniformierten SPOT-Kontrolleure, als schauten sie routinemäßig nach dem Rechten. Denjenigen, die sich gemessen an der Ekman'schen Checkliste sonderbar geben, weil sie etwa ängstlich, nervös oder verwirrt erscheinen oder Augenkontakte vermeiden, nähern sich die Fahnder, um deren Gesicht in einem kurzen, scheinbar unverfänglichen Gespräch näher nach solchen Mikro-Ausdrücken abzusuchen, in denen sich eine terroristische Planung verrät. Sobald sie fündig werden, ziehen die herbeigerufenen Autoritäten die üblichen Register (Personen- und Gepäckkontrolle usw.) gegenüber dem so als verdächtig ausgemachten Subjekt ${ }^{18}$.

Doch welche Emotionen erwartet man eigentlich kurz vor der Anschlagsausführung? Angst, Mitleid, Trauer oder Vorfreude auf das Jenseits? Von den TSA-Screenern wird das nicht preisgegeben - wohl, weil sie sich dieser Kriterien auch gar nicht gewiss sind. Wenn aber, wie hier, „die Gefahr nicht eindeutig identifiziert werden kann, dann vermag Prävention ihr nur vage zu begegnen. "19 So kam es in 2006 und 2007 zwar zu 43.000 SPOT-Gesprächen und 3100 Verdachtsfällen, doch ging ein mutmaßlicher Terrorist dabei mitnichten ins Netz. Überführt wurden lediglich 278 Personen - wegen Verletzung des Drogen-, Waffen- oder Einwandererrechts ${ }^{20}$. Immerhin könnte die Ekman-Group auch hier für Abhilfe sorgen. In einem neueren Projekt hat man sich den „signs of an immediate attack“ gewidmet und dabei einen „Dangerous Demeanor Detector (D-Cube)“ entwickelt, der jene Gesichtsausdrücke identifiziert, die charak- teristisch für die Vor-Attentats-Phase seien (etwa die sog. „Hinckley-Expression“) ${ }^{21}$.

So bliebe denn nur noch eine zweite, freilich sehr viel höhere Hürde: Man kann sich auf Menschen, und darin liegt eine Schwierigkeit aller "Surveillance“, oft nicht verlassen. Sie ermüden, sie sind abgelenkt, ihre Launen beeinflussen ihr Entscheidungsvermögen ${ }^{22}$. Der personalintensive Einsatz von SPOTKontrolleuren ist außerdem teuer. Das DHS präferiert deshalb eine automatisierte Erkennung der verdächtigen Mikroexpressionen und hat einem Team der „University of Buffalo“ die Entwicklung einer solchen Technologie anvertraut ${ }^{23}$. Das Vorhaben firmiert unter FAST: „Future Attribute Screening Technology“ (nachdem der ursprüngliche Arbeitstitel „Projekt Hostile Intent“ allzu verfänglich erschien). Herzstück des FAST-Aufbaus ist ein mit einer FACS-Software bestückter Computer, der eine unbegrenzte Zahl jener Gesichter analysiert, die eine versteckte Überwachungskamera bspw. beim Passieren einer Ausweiskontrolle oder in Wartezonen erfasst. Wird vom Apparat einen Antlitz bemerkt, das Anzeichen für böse Absichten zeigt, löst dies „manuelle“ Untersuchungen aus (SPOT-Gespräche, Kontrollen usw.). Weil ein Mikroausdruck allein aber noch keinen veritablen Terroristen ausmacht, wird das FACS-Gerät obendrein durch Geräte zur Erkennung vegetativer Prozesse flankiert: Angstzeichen wie eine plötzlich veränderte Körpertemperatur, ein hoher Blutdruck, eine gesteigerte Pulsfrequenz, schwere Atmung, kurz, all das, wofür sich auch ein Lügendetektor interessiert, wird durch ein sogenanntes „Bio-Lidar“ registriert. Sämtliche Geräte arbeiten verdeckt aus der Ferne und brauchen nicht länger, als die gefilmte Passabfertigung währt ${ }^{24}$. Darüber hinaus will man die Messanordnung bis 2012 durch einen Scanner zur Auswertung der Pupillenbewegung und zur Identifizierung der Iris, einen Sensor zur Analyse von Botenstoffen im Schweiß und einen Magnetresonanztomografen anreichern - alles, um weitere Lügeanzeichen und Enttarnungsängste aufzeichnen zu können.

Dieses Technikarsenal werde, so hieß es, den Amerikanern das ihnen abhandengekommene Freiheitsgefühl wiedergeben - mit FAST-Schleusen überall dort, wo dies angezeigt scheint (an Bahnhöfen, bei Sportveranstaltungen, Kundgebungen, Musikfestivals ... $)^{25}$. Ein erster Tauglichkeitstest fiel hierfür positiv aus: 4 von 5 Probanden, die einen verbotenen Gegenstand durch den Aufbau einer inszenierten Kontrolle schmuggeln und in der folgenden SPOT-Befragung verleug- nen sollten, wurden erkannt ${ }^{26}$. Doch was kann dieser Test schon erweisen? Aus gutem Grund wird nicht nur die Missbrauchbarkeit und Eingriffsschwere von FAST kritisiert, sondern mit Nachdruck bezweifelt, dass böse Gedanken unter Feldbedingungen gleichsam im Vorübergehen tatsächlich auslesbar sind. Stattdessen ist vom Gedankenscreening am Airport eine hohe Anzahl an false positives zu erwarten. Zeitnot, Flugangst, Vorfreude auf ein Wiedersehen, tränenreiche Abschiede, nörgelnde Kinder, wie will man den dadurch ausgelösten Normalstress von der Anspannung scheiden, die eine Terrorintention evoziert? Mikroexpressionen, die Verachtung, Angriffslust oder Furcht nahe legen, bringt gleichermaßen hervor, wer sich durch die Befragung der SPOT-Späher harangiert fühlt. Und die Angehörigen jener Nationalitäten, die de facto unter Generalverdacht stehen, weisen womöglich schon aus Angst vor dem üblichen Argwohn die suspekten Anzeichen auf ${ }^{27}$. Für das DHS sind dies freilich lässliche Schwächen. Der FAST-Pre-Crime-Detector gleiche einem gigantischen Netz, in dem sich zwar Delphine und andere harmlose Tierarten verheddern, doch rentiere sich dies wegen der Chance, den einen oder anderen Raubfisch zu fangen ${ }^{28}$.

\section{Die FAST-Sicherheit: Kontinuität und Qualitätssprung}

Warum sollten wir der FAST-Apparatur, sobald sie einen auch nur halbwegs ausgereiften Eindruck zu machen beginnt, nicht auch in Deutschland begegnen? Immerhin käme dies dem Bedarf an „Innerer Sicherheit" ersichtlich entgegen. Einer Terrorbedrohung, wie man sie hierzulande entwirft, ist mit der vorhandenen Erkennungstechnik nämlich nicht beizukommen. Die Experten der Gefahrenprognose rechnen mit der Abgefeimtheit der Angreifernetzwerke, gerade solche Personen ins Zielland zu senden, bei denen mangels Vorauffälligkeit die geläufigen Wiedererkennungs- und Alarmsysteme versagen. Wenn diesen Akteuren aber die polizeilichen Datensammlungen nichts anhaben können, ist man darauf angewiesen, ihren Akutzustand (samt ihrer böswilligen Absicht) rechtzeitig zu deuten. Die FASTSysteme, die Derartiges bieten, würden sich folglich als Gefahrenvorsorge leicht legitimieren (in Ansehung der verbreitet-dickfelligen „ich-habe-ja-nichts-zu-verbergen“Haltung allzumal).

Hielte diese Methode tatsächlich Einzug, stünde sie in einer bruchlosen Kontinuität zur eingangs skizzierten Vorermittlungs- 
Struktur: Denn FAST operiert heimlich ${ }^{29}$. Wer in die Wahrnehmung von Kamera und Sensorik gelangt, hat keine Ahnung, dass soeben seine Körperfunktion registriert und sein Gesichtsausdruck auf übelwollenden Vorsatz abgesucht wird. So wurden nicht einmal die Probanden des FAST-Experimentes gewahr, dass sie den Test absolvierten, weil die mobile Schleuse ihnen als neutraler Eingangsbereich erschien. FAST arbeitet ferner absolut anlasslos. Man installiert die Geräte auf eine vollkommen abstrakt bleibende Gefahr hin, die auf nichts weiter als der unbestimmten Erwartung irgendwo und irgendwann stattfindender Anschlagsereignisse gründet. Diese Anlasslosigkeit besteht sowieso gegenüber der unbescholtenen Person, aber auch gegenüber dem Passagier, der tatsächlich etwas verbirgt und womöglich sogar Terrorabsichten hegt. So liegt dem Gedankencheck schon deshalb kein Anfangsverdacht zugrunde, weil dafür Tatsachen mit zureichender Wahrscheinlichkeit auf eine tatsächlich begangene Straftat hindeuten müssten, wohingegen hier bis dato noch gar kein Delikt existiert. Und selbst wenn dem Reisenden objektiv ein strafbares Vorbereitungsgebaren vorwerfbar wäre ${ }^{30}$, existierte im maßgeblichen Zeitpunkt (d.h. unmittelbar bevor er gescreent wird) mangels irgendeines Argwohns noch kein konkreter Verdacht (weder gegen ihn noch gegen Dritte). Eben deshalb fehlt es im Übrigen auch an einer Polizeirechtsgefahr. Während deren gängige Deutung eine vertretbare ex-anteAnnahme sich abzeichnender Schadensverläufe verlangt (ohne auf die objektive Gefahrexistenz abzustellen) ${ }^{31}$, betreibt man die Schleuse in einer vagen, noch völlig unspezifizierten Terrorerwartung ${ }^{32}$. So gesehen wäre FAST ein typischer Fall der vorbeugenden Straftatbekämpfung ${ }^{33}$.

Gleichwohl nähme sich FAST nicht als Fortschreibung bestehenden Rechts, sondern als qualitativ neue und geradezu entgrenzte Eingriffsform aus. In der Gesichtslese-Schleuse trifft nämlich ein Zweifaches zusammen: Die Technik des Screenings und der $\mathrm{Zu}$ griff auf den Gedanken. Sicher, letzteres ist schon durchaus geläufig. Der Versuch, zur Verbrechensbekämpfung in das Verdächtigen-Denken vordringen zu wollen, zeigt sich bei der Vernehmung, in der man Habitus und Benehmen aufmerksam würdigt, ebenso wie in manchem psychologischen Test und erst recht beim Lügendetektor ${ }^{34}$. Auch mögen uns die Neurowissenschaften demnächst Hirnscanner bieten, von denen das Lügen oder die Persönlichkeitsstörung in bildgebenden Verfahren diagnostiziert werden $\mathrm{kann}^{35}$. All diese Verfahren haben mit den FAST-Geräten gemein, dass sie Körperindizien (Verhalten, Mimik, Herzschlag oder Hirnströme) systematisch verzeichnen, um hiervon interpretierend auf das Denken zu schließen. Bedenklich ist dies, weil es ins personale Zentrum abzielt, in den Ort, wo das Subjekt sich erst formt - und zwar vermittels von Regungen, über die ihr Produzent keine Herrschaft ausübt. Dies impliziert ein objekthaftes Verhältnis, in dem die eine Seite die unwillkürlichen Gedankenanzeiger der anderen Seite ausliest. Von daher werden solche Verfahren meist nur dort toleriert, wo man sie entweder gar nicht verbieten kann (wie bei der gesprächsimmanenten Lügenkontrolle) oder wo man sie dem Betroffenen nur mit dessen Einverständnis ansinnt (wie in der Regel bei den genannten Test). Doch: Bei FAST wird diese Dispositionsmacht von vornherein unterbunden! Der Passagier muss durch die Schleuse, ob er will oder nicht. FAST schaut jedem zustimmungsunabhängig hinter die Stirn. Und betroffen ist davon eine beinahe endlose Zahl. Indem sich das FAST-Verfahren auf eine enorme Menge unverdächtiger und auch nie verdächtig werdender Personen erstreckt ${ }^{36}$, kombiniert es die Methode der Gedankenenthüllung mit der Technik des auswahllosen, nicht abwendbaren Screenings der Massen ${ }^{37}$.

\section{Das Fazit: Apparate als Zeichen}

Zugegeben: Noch hat die Analyse (wenigstens hierzulande) etwas Spekulatives, und sie stößt, falls sich doch technologische (oder finanzielle) Hürden auftun, scheinbar ins Leere. Dass die geschilderte Anlage zur Wirklichkeit wird, ist aber gar nicht entscheidend. Entscheidend ist, dass ein solches Projekt gedacht, geplant und angepackt werden kann. Bereits dies macht FAST zu einem Zeichen der Sicherheitslogik, in dem die Vorsorge-Hybris sinnfällig wird. Es weist über die Video-Sensoren-ComputerApparatur folglich hinaus, wenn man angesichts entpersonalisierter Risikostrukturen ${ }^{38}$ einen Massenscanner ersinnt, der hinter all den zahllosen Gesichtern das feindselige Hirn isoliert und die "Terrorgefahr" damit re-personalisiert. Am Arsenal der Sensoren wird greifbar, dass es für das Sicherheitsdenken keine normativen Grenzen, sondern nur noch die Zufalls-Barrieren des technisch aktuell Machbaren gibt. Wer „das präventive Ende des liberaldemokratischen Rechtsstaats“39 vermerkt, stößt offensichtlich keine Augurenrufe hervor, sondern spricht begründete Warnungen aus. So würde der Einsatz der FAST-Totalausforschung in Deutschland wohl vermutlich auf keine le- gislativen Hemmungen stoßen - und auf das Veto des Verfassungsgerichts womöglich auch nicht (jedenfalls sollte man sich darauf nicht verlassen). Was wirklich wirken könnte, steht uns jedoch fortwährend vor Augen: die inszenierte Hysterie gegen den „Überwachungsstaat“ möglicherweise ...

Prof. Dr. Ralf Kölbel und Dr. Susanne Selter forschen und lehren Kriminologie und Strafrecht an der Universität Bielefeld.

\section{Literatur:}

Baumann, Zygmunt (2005): Verworfenes Leben. Die Ausgegrenzten der Moderne. Hamburg

Barrie, Allison (23.09.2008): Homeland Security detects terrorist threats by reading your mind, www.foxnews.com/story/0,2933,426485,00. html.

Boers, Klaus (1993): Kriminalitätsfurcht. Ein Beitrag zum Verständnis eines sozialen Problems. In: Monatsschrift für Kriminologie und Strafrechtsreform, S. $65-82$.

Bublitz, Hannelore (1999): Diskursanalyse als Gesellschafts-, ,Theorie“. In: dies. u. a. (Hrsg.): Das Wuchern der Diskurse, S. 22 - 48. Frankfurt a.M./New York.

Calgary, Herald (01.02.2008): www.canada.com/ calgaryherald/story.html?id=9ab9a6eb-78e14a6f-8581-fce2e.

Deleuze, Gilles (1993): Unterhandlungen 1972 - 1990. Frankfurt am Main.

Economist (23.10.2008): www.economist.com/ science/PrinterFriendly.cfm?story_id $=12$

Eisenberg, Ulrich (2005): Kriminologie, 6. Auflage. München.

Eisenberg, Ulrich (2008): Beweisrecht der StPO, 6. Auflage. München.

Ekman, Paul (2007): Gefühle Lesen. Heidelberg.

Ekman, Paul (2009): Telling Lies, Clues to Deceit in the Marketplace, 3. Auflage. Norton. Feeley, Malcom/Simon, Jonathan (1994): Actuarial Justice: The Emerging New Criminal Law. In: Nelken, David (Hrsg.), The Futures of Criminology, S. 173 - 201. London.

Frank, Mark (2008), Research Methods in Detecting Deception Research, In: Harrigan, Jinni / Rosenthal, Robert / Scheerer, Klaus (Hrsg.): The New Handbook of Methods in Nonverbal Behavior Research, S. 341 - 364. Oxford.

Frehsee, Detlev (2003): Der Rechtsstaat verschwindet. Gesammelte Aufsätze. Herausgegeben von Wolfgang Schild. Berlin.

Gamer (2008): Die Wahrheit über die Lüge. In: Gehirn \& Geist, Juli/August 2008, S. 33 $-37$.

Garland, David (2008): Kultur der Kontrolle, Verbrechensbekämpfung und soziale Ordnung in der Gegenwart. Frankfurt am Main.

Groenemeyer, Axel (2007): Gibt es eigentlich noch abweichendes Verhalten? Krisendiagnosen in Soziologie und Kriminologie. In: Kriminologisches Journal 39, S. $162-184$.

Haffke, Bernd (2005): Vom Rechtsstaat zum Sicherheitsstaat. In: Kritische Justiz, S. 17 - 35 .

Hassemer, Winfried (2006): Sicherheit durch Strafrecht. In: Strafverteidiger, S. 321 - 332.

Heath, Christian / Luff, Paul (2000): Technology in action, Cambridge.

Hefendehl, Roland (2000): Wie steht es mit der Kriminalitätsfurcht und was hat der Staat damit zu tun? In Kritische Justiz, S. $174-187$.

Hornung, Gerrit, (2008): Über Möglichkeiten und Grenzen der rechtlichen Bewertung neuer Überwachungstechnologien. In: Zurawski, 
Nils (Hrsg.): Surveillance Studies, S. 150 -166. Opladen.

Jasch, Michael / Hefendehl, Roland (2001): Kriminalgeographie und Furcht in ostdeutschen Städten. In: Monatsschrift für Kriminologie und Strafrechtsreform. S. $67-81$.

Kammerer, Dietmar (2008): Bilder der Überwachung, Frankfurt am Main.

Keller, Reiner (2005): Wissenssoziologische Diskursanalyse, Wiesbaden.

Kinzig, Jörg (2004): Die rechtliche Bewältigung von Erscheinungsformen organisierter Kriminalität. Berlin

Kölbel, Ralf (2006): Zur Problematik der strafprozessualen Körperhermeneutik. In: Goltdammers Archiv für Strafrecht, S. 469 - 492.

Krassmann, Susanne (2003): Die Kriminalität der Gesellschaft. Konstanz.

Kreissl, Reinhard (1997): Die Fake-Guerilla im Cybermarxismus, In: Frehsee, Detlev / Löschper, Gabi / Smaus, Gerlinda (Hrsg.): Konstruktion der Wirklichkeit durch Kriminalität und Strafe, S. 522 - 548. Baden-Baden.

Kunz, Karl-Ludwig (2004): Muss Strafe wirklich sein? Einige Überlegungen zur Beantwortung der Frage und zu den Konsequenzen daraus. In: Radtke, Henning u.a. (Hrsg.), Muss Strafe sein?, S. 71 - 83. Baden-Baden.

Lindenberg, Michael / Schmidt-Semisch, Henning (1995): Sanktionsverzicht statt Herrschaftsverlust. Vom Übergang in die Kontrollgesellschaft. In: Kriminologisches Journal 27, S. 2 $-17$.

Lisken, Hans / Denninger, Erhard (2007): Handbuch des Polizeirechts, 4. Aufl., München.

Löwe / Rosenberg (2003): Die Strafprozessordnung und das Gerichtsverfassungsgesetz. Großkommentar. Band 2, 25. Aufl., Berlin.

Narr, Wolf-Dieter (2009): Antiterroristische Mimesis - in Zeiten grenzenloser Prävention ein Abgrund an Sicherheit. In: Kriminologisches Journal 41, S. 2 - 19.

New Scientist (23.09.2008): www.newscientist.com/blogs/shortsharpscience/2008/09/ precrime-detector-is-s.

New York Times (28.04.2009): www.nytimes. com/2009/04/29/us/29surveil.html.

Prittwitz, Cornelius (1993): Strafrecht und Risiko, Frankfurt a.M.

Pütter, Norbert (1998): Der OK-Komplex, Münster.

Quensel, Stephan (2008): Der kulturelle Beitrag der Kriminologie zur hegemonialen Kontrolle. In: Monatsschrift für Kriminologie und Strafrechtsreform, S. 355 - 389.

Schleim, Stephan (2008): Lügen zwecklos. In: Gehirn \& Geist, Juli/August 2008, S. 40 - 46.

Seattle Times (20.09.2007): http://seattletimes.nwsource.com/html/nationworld/2003893183_ tsa200.html

Singelnstein, Tobias (2007): Jeder ist verdächtig. Das neue gesellschaftliche Verständnis von Sicherheit und Bedrohung als Grundlage sozialer Kontrolle. In: vorgänge - Zeitschrift für Bürgerrechte und Gesellschaftspolitik, S. 118 $-126$.

Singelnstein, Tobias / Stolle, Peer (2008): Die Sicherheitsgesellschaft, 2. Aufl., Wiesbaden.

Time Magazine (20.08.2006): www.time.com/ time/magazine/article/0,9171,1229109,00. html.

Time Magazine (17.05.2006): www.time.com/nation/article/0,8599,1195330,00.html.

Transport Security Administration (2009): Where we stand, TSA trains hard for new threats, www.tsa.gov/press/where_we_stand/training. shtm.

U.S. Department of Homeland Security (2008): Future Attribute Screening Technology Project, www.dhs.gov/xlibrary/assets/privacy/ privacy_pa_st_fast.pdf. van Elsbergen, Gisbert (2008): Kriminologische Implikationen der Videoüberwachung. In Zurawski Nils (Hrsg.): Surveillance Studies, S. 104 - 117, Opladen.

Weßlau, Edda (1998): Vorfeldermittlungen, Berlin.

Wild, Jennifer (2005): MRI Scans can pick up lies, but raise ethical issues, In: Nature 437, S. 457.

Wolter, Jürgen (2004): Potential für eine Totalüberwachung im Strafprozess- und Polizeirecht. In: Rogall, Klaus (Hrsg.), Festschrift für Hans-Joachim Rudolphi zum 70. Geburtstag, S. $733-748$. Neuwied.

Zöller, Mark Alexander (2002): Informationssysteme und Vorfeldmaßnahmen von Polizei, Staatsanwaltschaft und Nachrichtendiensten, Heidelberg.

\section{Fußnoten:}

1 Allgemein zur Kriminalitätsfurcht: BOERS (1993); HEFENDEHL (2000); JASCH / HEFENDEHL (2001).

2 Eingehend SINGELNSTEIN / STOLLE (2008); GARLAND (2008); HASSEMER (2006); HORNUNG, (2008); KAMMERER (2008); NARR (2009); VAN ELSBERGEN (2008).

3 Vgl. HAFFKE (2005), S. 20 ff.

4 Dazu HASSEMER (2006), S. 326; ZÖLLER (2002), S. 132.

5 Zur diskursiven Konstruktion von Risiken vgl. KELLER (2005), S. 183 ff.; KUNZ (2004), S. 71 f; BUBLITZ (1999), S. 23 f, 31; FREHSEE (2003) S. 399 f.; SINGELNSTEIN (2007), S. $119 \mathrm{f}$.

6 Vgl. KRASSMANN (2003), S. 108 ff.; GROENEMEYER (2007), S. $162 \mathrm{ff}$.

7 Vgl. FEELEY / SIMON (1994), S. 173 ff.; LINDENBERG / SCHMIDT-SEMISCH (1995), S. 3. Und zugleich werden von diesem Diskurs i.Ü. „die Produktionsfaktoren der Risiken (...) ausgespart" (NARR, 2009, S. 4).

8 Dazu, dass der Staat das tut, vgl. BAUMANN (2005). Ein Bsp. bieten die Vorverlagerungstendenzen im materiellen Strafrecht (Risikoneutralisierung durch Gefährdungstatbestände). Dazu PRITTWITZ (1993).

9 Vgl. CASTLE (2005), S. 9 ff.; QUENSEL (2008), S. 355 , S. 369 f.

10 DELEUZE (1993), S. 254.

11 Vgl. z.B. $\$ \mathbb{S} 22,23 a, 36$ I Nr. 2 PolG BW oder $\mathbb{\$} 1 \mathrm{~V}, 14$ ff., 20 I Nr. 2, 21 I PolG NW.

12 Vgl. KINZIG (2004), S. 87 ff.; PÜTTER (1998), S. 12 f.; WOLTER (2004), S. 733 ff.; EISENBERG (2005), $\$ 27$, Rdnr. 34 ff. zu den genannten Strukturmerkmalen. Speziell zur Grundrechtswesentlichkeit von Vorfeldermittlungen vgl. BVerfGE 115, S. 320 ff. (Rasterfahndung); BVerfGE 118, S. 168 ff. (Kontostammdaten); BVerfGE 120, S. 378 ff. (automatisierte KfzKennzeichen-Abfrage).

13 Näher dazu und zum Niederschlag, den diese Körperhermeneutik im historischen und gegenwärtigen Strafprozess gefunden hat, vgl. KÖLBEL (2006).

14 Die von Ekman entwickelte Technik des Gesichterlesens ist beschrieben in EKMAN (2007); für einen Überblick: http://www.scholarpedia.org/ article/Facial_expression_analysis.

15 Zur Lügeerkennung anhand lügebegleitender Emotionsausdrücke vgl. EKMAN (2009); zuletzt etwa FRANK (2008) und zusammenfassend KÖLBEL (2006), S. $486 \mathrm{f}$.

$16 \mathrm{Zu}$ den Mikroexpressionen vgl. EKMAN (2009) sowie www.paulekman.com.

17 Hierzu und zum Folgenden vgl.: TRANSPORT SECURITY ADMINISTRATION (2009); TIME MAGAZINE (17.05.2006).

18 TRANSPORT SECURITY ADMINISTRATION (2009); TIME MAGAZINE (17.05.2006)
19 NARR (2009), S. 5.

$20 \mathrm{Vgl}$. TRANSPORT SECURITY ADMINISTRATION (2009); CALGARY HERALD (01.02.2008)

21 Benannt nach dem Reagan-Attentäter John Warnock Hinckley, dessen prädeliktische Videoaufzeichnung (ebenso wie die anderer Terroristen) ausgewertet wurde. Zum Ertrag dieser Forschung: www.dangerousdemeanor.com.

22 Instruktive Hinweise für den Fall der Videoüberwachung bei HEATH/LUFF (2000), S. 88 ff.

23 Beschreibung des Forschungsauftrags bei: U.S DEPARTMENT OF HOMELAND SECURITY (2008). Ekman ist gegenüber diesem (!) Vorhaben offenbar skeptisch.

24 Zur genauen Funktionsweise der aufgeführten Geräte vgl. TIME MAGAZINE (20.08.2006).

25 BARRIE (23.09.2008), S. 3.

$26 \mathrm{Zu}$ den in Maryland durchgeführten FAST-Tests vgl. NEW SCIENTIST (23.09.2008); ECONOMIST (23.10.2008).

27 SEATTLE TIMES (20.09.2007); NEW YORK TIMES (28.04.2009). Vgl. i.Ü. auch BVerfGE 115, S. 320, S. 339, 351 zur Rolle von Vorurteilen bei der Rasterfahndung.

28 Hierzu U.S. DEPARTMENT OF HOMELAND SECURITY (2008).

29 Zur rechtsstaatlichen Bedenklichkeit heimlicher Informationserhebung z.B. BVerfGE 118, 168 197; BVerfGE 120, 378, 403, 406.

30 Dafür wird durch das moderne Vorfeldstrafrecht (Anm. 8) meistens gesorgt, etwa in $\mathrm{Ge}-$ stalt der neuen Terrorbekämpfungsvorschriften ( $\$ \$ 89$ a, 89 b, 91 StGB-E) oder der $\$ \$ 30,127$ 129 f., 130 a StGB.

31 Vgl. etwa Denninger in: LISKEN / DENNINGER (2007), E 47, zum subj. Gefahrbegriff der h.M.

32 Auch ein Gefahrenverdacht (dazu Denninger in: LISKEN /DENNINGER (2007), E 48) liegt hier deshalb nicht vor.

33 Selbst Vorfeldermittlungen setzen nach h.M freilich Umstände voraus, nach denen gemäß allgemeiner Lebenserfahrung gepaart mit polizeilichem Fachwissen die künftige Begehung von Straftaten zu erwarten ist. Vgl. etwa WEßLAU (1998), S. 335 f.; ZÖLLER (2002), S. 82. Im Falle von FAST müsste man den Airport dafür als generell gefährdetes Feld deklarieren.

34 Vgl. nur EISENBERG (2008), Rdnrn. 693 ff., $1619 \mathrm{~g}, 2312 \mathrm{f}$.

$35 \mathrm{Zu}$ diesen neurowissenschaftlichen Verheißungen einführend SCHLEIM (2008), S. 40 ff.; für einen ersten Überblick zur Lügeerkennung durch Kernspintomografie vgl. WILD (2005); GAMER (2008) und www.luegendetektion.de.

36 Gemessen daran, dass FAST in seiner „Eingangsstufe" zahllose Unverdächtige tiefgehen inspiziert, ist es sekundär, ob der Scanner dann Falschverdächtigungen generiert (oben bei Fn 27), denn selbst bei einer $100 \%$ igen Trefferquote stellt sich die Frage, ob man hierfür scharenweise Unbeteiligten ins Hirn schauen darf. Zur besonderen Eingriffsqualität von verdachtslosen und mit großer Streubreite versehenen Überwachungsmaßnahmen vgl. BVerfGE 115 , $320,354 \mathrm{ff}$

37 Das verdient unterstrichen zu werden, weil man die In-Beschlagnahme Unverdächtiger durch Screening-Methoden üblicherweise mit der $\mathrm{Ge}$ ringfügigkeit des Eingriffs legitimiert, der für sie „keine spürbaren Belastungen" mit sich bringe. So für die Rasterfahndung Schäfer in LÖWE / ROSENBERG (2003), \$ 98a Rdn. 14.

38 Oben vor Fn. 7.

39 So - stellvertretend (!) - NARR (2009), S. 13. 